\title{
The impact of hemoglobin on the efficacy of phototherapy in hyperbilirubinemic infants
}

\author{
Mette L. Donneborg ${ }^{1}$, Pernille K. Vandborg ${ }^{1}$, Bo M. Hansen ${ }^{2}$, Maria Rodrigo-Domingo ${ }^{3}$ and Finn Ebbesen ${ }^{1}$
}

BACKGROUND: Phototherapy is the routine treatment for neonatal hyperbilirubinemia. Absorption of light in the skin transforms the native Z,Z-bilirubin to photobilirubins. This study investigates whether the hemoglobin concentration has an impact on efficacy of phototherapy, expressed by the decline of total serum bilirubin concentration (TsB).

METHODS: A trial was conducted on 93 infants, gestational age $\geq 33$ weeks, with uncomplicated hyperbilirubinemia. The infants were treated with conventional phototherapy using LED light for $24 \mathrm{~h}$. The median light irradiance was $66.8 \mu \mathrm{W} /$ $\mathrm{cm}^{2} / \mathrm{nm}$.

RESULTS: The median decrease in TsB after $24 \mathrm{~h}$ was 121 (57199) $\mu \mathrm{mol} / \mathrm{/}$; the median hemoglobin was 12.0 (7.0-14.7) $\mathrm{mmol} / \mathrm{l}$. There was a significant effect of hemoglobin concentration on the decrease in TsB of $-3.61 \mu \mathrm{mol} / \mathrm{mmol}$ hemoglobin $(P=0.022)$, after adjusting for initial TsB and postnatal age. That is, assuming the same initial TsB and postnatal age, for each $\mathrm{mmol} / \mathrm{l}$ increase in hemoglobin, the decrease in TsB was $3.61 \mu \mathrm{mol} / / \mathrm{l}$ smaller. In our hemoglobin range, the decrease in TsB is reduced by $28 \mu \mathrm{mol} / \mathrm{I}(23 \%)$.

CONCLUSION: Increasing hemoglobin levels led to a decrease in the efficacy of phototherapy. Our data provide additional support for the conclusion that the transformation of bilirubin to photobilirubins takes place mainly in the superficial capillaries of the skin.

J aundice in neonates is common, with $2-6 \%$ of infants requiring treatment for this condition. Over the last 50 years, phototherapy has been used to treat neonatal hyperbilirubinemia to prevent extremely high levels of bilirubin that may cause bilirubin-induced encephalopathy. The treatment has improved as the light sources and strategy for using these have been optimized. Exchange transfusions have been increasingly replaced by phototherapy because of both the efficacy and the superior safety profile of the latter (1).

It is well known that phototherapy transforms unconjugated bilirubin from the native, non-polar, and neurotoxic Z,Z-bilirubin to more polar isomers called photobilirubins that consists of configurational isomers Z,E- and E,Z-bilirubin and structural isomers E,Z- and E,E-lumirubin (2). These isomers can then be excreted without conjugation into the bile and the structural isomers into the urine (3). Whether the biochemical processes during phototherapy occur predominantly intravascularly or extravascularly has long been debated, although recent evidence strongly supports the idea that the biochemical processes during phototherapy predominantly take place intravascularly. In these in vitro studies, it has also been found that hemoglobin is a major competitor with bilirubin for the absorption of light during light exposure $(4,5)$. The authors predicted that a high hematocrit would reduce the therapeutic efficacy of phototherapy; however, this has never been confirmed in vivo $(4,5)$. Confirming this hypothesis in a clinical setting is an important step in understanding the basic mechanisms of phototherapy, and could have implications for the treatment strategy for neonates with hyperbilirubinemia.

In the present clinical study, we investigated whether the efficiency of phototherapy, expressed as the decline in the total serum bilirubin concentration (TsB), is dependent on the concentration of hemoglobin. We hypothesized that the efficacy of phototherapy will decrease with increasing hemoglobin concentrations.

\section{METHODS}

A prospective trial was conducted between 15 June 2014 and 15 June 2015 in the neonatal intensive care unit at Aalborg University Hospital, Denmark. Inclusion criteria were otherwise healthy term or late preterm (gestational age $\geq 33$ weeks) newborn infants with hyperbilirubinemia, without signs of hemolytic disease, birth weight $\geq 1,800$ g, postnatal age $>24 \mathrm{~h}$ and $\leq 7$ days, who had not previously received phototherapy, and who could be treated in a crib. Infants fulfilling the indications for exchange transfusion or double phototherapy due to a very high initial or rapidly increasing TsB were not enrolled. The indications for phototherapy followed the existing guidelines of the North Denmark Region.

The attending physician enrolled the infants consecutively in the study. One hundred infants were eligible for the study. The parents of six of the infants did not wish to participate, and one infant was excluded from the study because of Rhesus isoimmunization. Thus, the study cohort consisted of 93 infants who received phototherapy with blue LED light from above at a distance of $30 \mathrm{~cm}$ between the light source and the mattress. The distances between the lamps and

${ }^{1}$ Department of Pediatrics, Aalborg University Hospital, Aalborg, Denmark; ${ }^{2}$ Department of Pediatrics, Herlev Hospital, Copenhagen, Denmark; ${ }^{3}$ Unit of Epidemiology and Biostatistics, Aalborg University Hospital, Aalborg, Denmark. Correspondence: Mette L. Donneborg (mld@rn.dk)

Received 24 April 2017; accepted 31 July 2017; advance online publication 6 September 2017. doi:10.1038/pr.2017.186 


\section{Articles | Donneborg et al.}

the mattress were adjusted with a wood template for each infant. The average distance from the light source to the surface of the infant was $21.5 \mathrm{~cm}$.

All infants received phototherapy for $24 \mathrm{~h}$. They were naked except for diapers and eye pads. Phototherapy was administered continuously except during 30 min of feeding and nursing care every third hour. Previous studies have shown that the infants received phototherapy $85 \%$ of the time on average with this regime (6). The weight of the infants was registered before and after phototherapy.

\section{Measurements}

The phototherapy device was the neoBLUE LED phototherapy system (Natus Medical, San Carlos, CA) that emits blue light in the $420-520 \mathrm{~nm}$ spectrum, centered at $458 \mathrm{~nm}$ with a bandwidth of $450-470 \mathrm{~nm}$, which is defined as the spectral range at half peak irradiance. Irradiance was measured with a neoBLUE radiometer (Natus Medical), which measures spectral irradiance from 420 to $500 \mathrm{~nm}$ with maximum sensitivity in the spectrum of $440-480 \mathrm{~nm}$. The device was calibrated just before initiation of the study.

During phototherapy, irradiance was measured three times, approximately every eighth hour, at the infants' head, abdomen, and knees, and the mean value was calculated. Because of the height of the radiometer, these measurements were obtained $3.5 \mathrm{~cm}$ above the infant, which results in higher levels of irradiance than at the skin level. The ratio between the irradiance at the skin level and the measured irradiance was 0.93 . This correction factor was employed.

The irradiance of the phototherapy device was measured in a footprint containing the illuminated area measuring $21 \times 28 \mathrm{~cm}$. It was measured in a grid consisting of 12 squares of $7 \times 7 \mathrm{~cm}, 8.5 \mathrm{~cm}$ above the mattress level, corresponding to the average height over the mattress of the exposed skin of the infants. The mean irradiance of the footprint was $64.8 \mu \mathrm{w} / \mathrm{cm}^{2} / \mathrm{nm}$. We also measured the irradiance in a bigger illuminated area measuring $28 \times 49 \mathrm{~cm}$, in a grid consisting of 28 squares of $7 \times 7 \mathrm{~cm}$ (ref. 7). The mean irradiance was $53.1 \mu \mathrm{w} / \mathrm{cm}^{2} /$

Table 1. Baseline demographic, clinical, and irradiance data

\begin{tabular}{lc}
\hline Infants $(n)$ & 93 \\
Gender: female/male $(n)$ & $45 / 48$ \\
Non-Caucasian ethnicity $(n)$ & $9^{\mathrm{a}}$ \\
Age & \\
Gestational age (days) & $269(239-293)$ \\
Postnatal age $(\mathrm{h})^{\mathrm{b}}$ & $78(41-164)$ \\
& \\
Weight & \\
Birth weight $(\mathrm{g})^{\mathrm{b}}$ & $3,270(1,835-5,090)$ \\
Weight change from birth to phototherapy $(\%)^{\mathrm{b}}$ & $-4.7(-10.8-2.6)$ \\
Weight change during phototherapy $(\%)^{\mathrm{b}}$ & $0.6(-3.8-7.6)$
\end{tabular}

Feeding during phototherapy ( $\mathrm{n}$ )

Exclusively breast-fed

39

Exclusively formula-fed

6

Mixed formula/breast-fed

48

Light irradiance, measured $\left(\mu \mathrm{W} / \mathrm{cm}^{2} / \mathrm{nm}\right)^{\mathrm{b}, \mathrm{c}}$

$71.8(48.9-84.0)$

Adjusted light irradiance $\left(\mu \mathrm{W} / \mathrm{cm}^{2} / \mathrm{nm}\right)^{\mathrm{b}, \mathrm{d}}$

$66.8(45.5-78.1)$

aSeven Asian, one African, and one Middle Eastern.

bedian (range).

'Light irradiance: mean of all three measurements for each infant.

${ }^{\mathrm{d}}$ The irradiance was measured $3.5 \mathrm{~cm}$ above the infant because of the dimensions

of the light sensor. A correction factor of 0.93 was employed. $\mathrm{nm}$. The levels of irradiance did not change during the study.

$\mathrm{TsB}$ was measured at the start $\left(\mathrm{TsB}_{0}\right)$ and after $24 \mathrm{~h}\left(\mathrm{TsB}_{24}\right)$ of treatment in capillary blood drawn by a heel stick. It was analyzed at the Department of Clinical Biochemistry, Aalborg University Hospital, with the diazo method using Cobas 8000 (Roche Diagnostics International, Mannheim, Germany) (8). The imprecision expressed as coefficient of variation was 3.0 and $1.4 \%$ at TsB levels of 176 and $333 \mu \mathrm{mol} / 1$, respectively. External quality assessment showed a bias below $5 \%$ in the interval 10-1,000 $\mu \mathrm{mol} / \mathrm{l}$ (7).

Hemoglobin was measured in the capillary blood at the start of phototherapy, drawn concurrently with $\mathrm{TsB}_{0}$. It was analyzed at the Department of Clinical Biochemistry, Aalborg University Hospital with the photometric method using ABL (Radiometer, Copenhagen, Denmark). The imprecision expressed as coefficient of variation was $<0.5 \%$ in the hemoglobin range $5-12 \mathrm{mmol} / \mathrm{l}$, and generally the variation was smaller at higher concentrations (validated based on control material from Radiometer, at the Department of Clinical Biochemistry, Aalborg University Hospital).

Information was registered about gender, gestational age, ethnicity, birth weight, postnatal age, and feeding type for the infants.

\section{Ethics}

The Regional Committee on Health Research Ethics in North Denmark Region approved the study. Verbal and written informed consent was obtained from the parents of the infants. The study was registered at ClinicalTrials.gov.

\section{Statistical Analysis}

On the basis of a pilot study $(n=22)$, at least 90 infants were required to detect an effect size of $8 \mu \mathrm{mol} / \mathrm{l}$ decrease in TsB per $1 \mathrm{mmol} / \mathrm{l}$ increase in hemoglobin. The SDs used were $1.2 \mathrm{mmol} / \mathrm{l}$ for hemoglobin and $30 \mu \mathrm{mol} / \mathrm{l}$ for decrease in TsB. This sample size was sufficient to achieve $80 \%$ power with a significance level of $\alpha=0.05$ in an unadjusted linear regression.

$\mathrm{TsB}_{0}, \mathrm{TsB}_{24}$, and change in TsB in $\mu \mathrm{mol} / \mathrm{l}$ after $24 \mathrm{~h}$ of treatment $\left(\Delta \mathrm{TsB}_{0-24}\right)$ as well as hemoglobin concentration are presented as the medians with their respective ranges.

Simple linear regression analyses were performed with $\Delta \mathrm{TsB}_{0-24}$ as the response variable and hemoglobin, $\mathrm{TsB}_{0}$, gestational age, birth weight, postnatal age, infants receiving formula (exclusively or as supplementation), and light irradiance as explanatory variables. The explanatory variables that were statistically significant in each of the simple linear regressions were included in a multivariable linear regression. Besides, a sensitivity analysis was carried out to investigate the effect of outliers.

Statistical analyses were performed in Stata 13 (StataCorp, College Station, TX, USA). Results with $P$-values below 0.05 were considered statistically significant.

\section{RESULTS}

Baseline demographic and clinical data of the 93 infants are presented in Table $1 . \mathrm{TsB}_{0}, \mathrm{TsB}_{24}, \Delta \mathrm{TsB}_{0-24}$, and hemoglobin concentrations are presented in Table 2. As shown, the median TsB decrease was $121 \mu \mathrm{mol} / \mathrm{l}$ after $24 \mathrm{~h}$ of phototherapy. Hemoglobin ranged from 7.0 to $14.7 \mathrm{mmol} / \mathrm{l}$, median $12.0 \mathrm{mmol} / \mathrm{l}$.

The regression analyses are presented in Table 3 . The simple linear regression analyses showed that the following variables had a significant impact on $\Delta \mathrm{TsB}_{0-24}$ : hemoglobin, $\mathrm{TsB}_{0}$, and postnatal age. The multivariable regression model therefore included these three statistically significant parameters. $\Delta \mathrm{TsB}_{0-}$ 24 was associated negatively with the hemoglobin concentration and positively with $\mathrm{TsB}_{0}$ and postnatal age. The estimated regression coefficient for hemoglobin was $-3.61(P=0.022)$. 
Assuming a postnatal age of $78 \mathrm{~h}$ and an initial TsB of $270 \mu \mathrm{mol} / \mathrm{l}$, the infant with a hemoglobin concentration of $7.0 \mathrm{mmol} / \mathrm{l}$ would experience a $140 \mu \mathrm{mol} / \mathrm{l} \mathrm{TsB}$ decrease compared with a $112 \mu \mathrm{mol} / \mathrm{l}$ decrease for the infant with a hemoglobin concentration of $14.7 \mathrm{mmol} / \mathrm{l}$. That is, a decrease of $28 \mu \mathrm{mol} / \mathrm{l}$ in $\mathrm{TsB}$ within the observed hemoglobin range. These observed maximum and minimum hemoglobin values might not be considered normal in a clinical setting. Therefore, we chose to look at the difference in effect between 9.0 and $14.0 \mathrm{mmol} / \mathrm{l}$, which are the $5 \%$ and the $95 \%$ percentiles of hemoglobin. The decrease in TsB was $18 \mu \mathrm{mol} / \mathrm{l}$ smaller at the upper end of this hemoglobin range than at the lower end.

Scatter plots of change in TsB vs. hemoglobin concentration are presented in Figure 1. The figure shows both the unadjusted regression line, and the regression line adjusted for $\mathrm{TsB}_{0}$ and postnatal age in hours. At any hemoglobin level, there was a large variation in the decrease of TsB during the treatment as seen in the scatter plot.

A sensitivity analysis was performed excluding the infant with a hemoglobin concentration of $7.0 \mathrm{mmol} / \mathrm{l}$. It only had a minor influence on the results; the hemoglobin coefficient was $-5.82(P=0.007)$ in the simple regression and -3.47 $(P=0.036)$ in the multivariable regression.

No side effects of phototherapy other than loose stools were observed. No infants had hypo- or hyperthermia, and no rashes were seen.

\section{DISCUSSION}

Table 2. Changes in total serum bilirubin concentration after $24 \mathrm{~h}$ of phototherapy, and hemoglobin concentration at initiation of phototherapy

\begin{tabular}{|c|c|}
\hline $\mathrm{TsB}_{0}, \mu \mathrm{mol} / \mathrm{I}^{\mathrm{a}}$ & $270(181-338)$ \\
\hline $\mathrm{TsB}_{24}, \mu \mathrm{mol} / \mathrm{I}^{\mathrm{a}}$ & $146(52-209)$ \\
\hline$\Delta \mathrm{TsB}_{0-24}, \mu \mathrm{mol} / \mathrm{I}^{\mathrm{a}}$ & 121 (57-199) \\
\hline Hemoglobin, $\mathrm{mmol} / \mathrm{l}^{\mathrm{a}}$ & $12.0(7.0-14.7)$ \\
\hline \multicolumn{2}{|c|}{$\begin{array}{l}\mathrm{Ts}_{0} \text {, total serum bilirubin concentration at the start of phototherapy; } \mathrm{TsB}_{24} \text {, total } \\
\text { serum bilirubin concentration after } 24 \mathrm{~h} \text { of phototherapy; } \triangle \mathrm{TsB}_{0-24} \text {, } \\
\text { difference between } \mathrm{TsB}_{0} \text { and } \mathrm{TsB}_{24} \text {. } \\
\text { a Median (range). }\end{array}$} \\
\hline
\end{tabular}

Our overall results showed that the efficacy of phototherapy was significantly dependent on the concentration of hemoglobin. For every $1.0 \mathrm{mmol} / \mathrm{l}$ increase in hemoglobin concentration, the decrease in TsB during $24 \mathrm{~h}$ of phototherapy was $3.61 \mu \mathrm{mol} / \mathrm{l}$ smaller. To our knowledge, this is the first prospective clinical study corroborating that hemoglobin is a competitor with bilirubin in phototherapy, an observation made in in vitro studies.

In 1983, Granati et al. (9) showed in vitro that, as hematocrit declined, bilirubin photodegradation increased; however, the correlation was not confirmed in vivo. In another in vitro study, by Lamola et al. (4), using a semiempirical skin model, it was confirmed that hemoglobin in the skin competes with bilirubin for absorption of light during phototherapy. This was strongest at the wavelength 400$460 \mathrm{~nm}$, also found in vitro by Linfield et al. (5) in 2016. On the basis of these results, it was predicted that a high hematocrit would reduce the therapeutic efficacy of phototherapy in vivo.

From a theoretical perspective, there is increasing, although indirect, evidence that isomerization of bilirubin in vivo primarily occurs in the skin capillary circulation $(10,11)$. Our

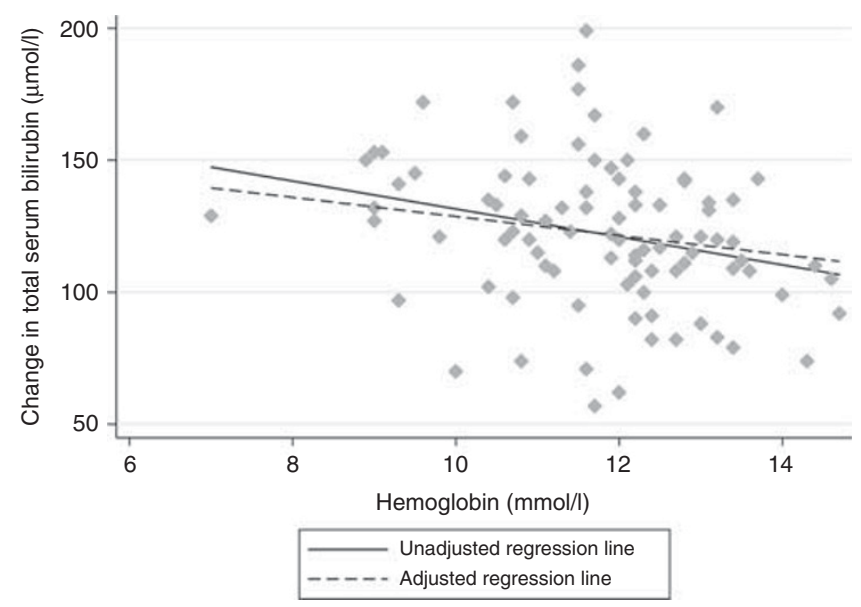

Figure 1. Scatter plots of change in total serum bilirubin concentration during $24 \mathrm{~h}$ of phototherapy vs. hemoglobin concentration.

Table 3. Regression analyses

\begin{tabular}{|c|c|c|c|c|}
\hline \multirow[t]{2}{*}{ Explanatory variables } & \multicolumn{2}{|c|}{ Simple linear regressions } & \multicolumn{2}{|c|}{ Multivariable linear regression } \\
\hline & Coefficient $(95 \% \mathrm{Cl})$ & $P$ value & Coefficient $(95 \% \mathrm{Cl})$ & $P$ value \\
\hline Hemoglobin, mmol/l & $-5.29(-9.20,-1.38)$ & 0.009 & $-3.61(-6.69,-0.53)$ & 0.022 \\
\hline Gestational age, days & $0.25(-0.16,0.66)$ & 0.23 & & \\
\hline Birth weight, kg & $-1.76(-10.65,7.13)$ & 0.70 & & \\
\hline Irradiance, $\mu \mathrm{W} / \mathrm{cm}^{2} / \mathrm{nm}$ & $-0.10(-0.94,0.74)$ & 0.81 & & \\
\hline
\end{tabular}

$\mathrm{Cl}$, confidence interval; $\mathrm{TsB}_{0}$, total serum bilirubin concentration at the start of phototherapy; $\mathrm{Ts}_{24}$, total serum bilirubin concentration after $24 \mathrm{~h}$ of phototherapy; $\triangle \mathrm{TsB}_{0-24}$, difference between $\mathrm{TsB}_{0}$ and $\mathrm{TsB}_{24}$ 


\section{Articles | Domnebors et $t$ al.}

findings support this understanding. Previously, the clinical observation that the yellow color of the skin in the jaundiced infant diminishes during the first hours of phototherapy had led to the understanding that transformation of Z,Z-bilirubin to photobilirubins mainly takes place extravascularly in the skin. Pursuant to this understanding, infants in phototherapy were routinely alternated from prone to supine position to increase the efficacy of phototherapy (12). However, this routine was largely discontinued when it was found that the efficacy of phototherapy is independent of alternating the position of the infant $(10,13-15)$. Other observations also support the hypothesis that the effect of phototherapy is predominantly intravascular. Thus, $15 \mathrm{~min}$ after phototherapy is initiated, a substantial amount of the photobilirubin Z,Ebilirubin can be measured in plasma, corresponding to $\sim 10 \%$ of total bilirubin (11). This finding seems inconsistent with diffusion from the extravascular to the intravascular compartment, as diffusion is a much slower process.

Our finding of an inverse relationship between hemoglobin and the efficacy of phototherapy could be explained by an intravascular effect of phototherapy on bilirubin isomerization (4). The hemoglobin in the erythrocytes will absorb light, which implies that a fraction of the light will not reach the bilirubin molecules, i.e., hemoglobin in the erythrocytes will compete with the bilirubin molecules and thereby influence the efficacy of phototherapy. This has also been investigated by measuring the photobilirubins. The formation of Z,E-bilirubin is reversible, and Z,E-bilirubin accumulates rapidly in plasma at commonly used levels of irradiance because of a fast formation and a slow excreting rate. Within a few hours of phototherapy, the ratio of Z,E-bilirubin to Z,Zbilirubin reaches a plateau, which means that a photoequilibrium occurs between these two substances (16). Mreihil et al. (11) in 2015 compared the rate and level of $4 \mathrm{Z}, 15 \mathrm{E}$ photoisomerization during intensive phototherapy and did a post hoc analysis of hemoglobin data from 36 of their patients. They found a significant, negative correlation between hemoglobin concentration and percentage of Z,E-bilirubin after 15, 30, and $60 \mathrm{~min}$; however, it disappeared at the later time points. This was explained by the fact that when an equilibrium between Z,Z-bilirubin and Z,E-bilirubin is obtained in plasma, the percentage of Z,E-bilirubin only depends on the emission spectrum of the light, and is independent of the irradiance. The authors concluded that the hemoglobin concentration would influence the efficacy of phototherapy until an equilibrium between Z,Z- and Z,E-bilirubin occurs.

However, we found an effect of hemoglobin on the bilirubin decline in plasma after $24 \mathrm{~h}$ of phototherapy. We suggest that this is explained by the fact that the production of E,Zlumirubin is the most important bilirubin-lowering process during intensive phototherapy, and an equilibrium between $E$, Z-lumirubin and Z,Z-bilirubin does not occur $(3,16,17)$. Therefore, the decline of bilirubin is dependent on hemoglobin concentration throughout the whole treatment period and not just during the first $60 \mathrm{~min}$, as shown by Mreihil et al. (11).
From a clinical perspective, our results may be beneficial for clinicians treating hyperbilirubinemic infants with phototherapy. We have included infants with gestational ages ranging from 33 to 41 weeks; the mean hemoglobin concentration increases in this period of gestation from 10.4 to $11.5 \mathrm{mmol} / \mathrm{l}$ (ref. 18). Over a period of $24 \mathrm{~h}$, the average decrease in TsB will be $\sim 18 \mu \mathrm{mol} / \mathrm{l}$ less in infants with a hemoglobin concentration of $14.0 \mathrm{mmol} / \mathrm{l}$ than in infants with a hemoglobin concentration of $9.0 \mathrm{mmol} / \mathrm{l}$, which is equivalent to a decrease of $15 \%$ less. Thus, infants with high hemoglobin concentration are likely to need a longer duration of treatment, higher irradiance, or a larger body-surface area exposed to phototherapy, compared with infants with a lower hemoglobin concentration, in order to achieve the same decrease in TsB. This could be important for the decision of when to draw the next blood sample. An algorithm predicting the effect of phototherapy should probably include hemoglobin concentration. In a "crash cart" case where the level of TsB should be reduced as fast as possible, the influence of the variation in the hemoglobin level on the decrease of TsB during phototherapy might be considered as minimal.

Our study has some limitations. The bilirubin production rate was not measured directly as the end expiratory excretion rate of CO (CoSense ${ }^{\mathrm{R}}$, CAPNIA, Redwood City, CA) because this method was not available at the start of the study.

Another limitation is that a higher hemoglobin level may be associated with a higher bilirubin production rate appearing as a higher rate of bilirubin increase in plasma. The shown negative relationship between $\Delta \mathrm{TsB}_{0-24}$ and hemoglobin could be explained by a higher rate of bilirubin production at higher hemoglobin concentrations. However, $\Delta \mathrm{TsB}_{0-24}$ was adjusted for $\mathrm{TsB}_{0}$ and postnatal age. $\mathrm{TsB}_{0}$ in relation to postnatal age is the increase in TsB from birth to the start of phototherapy, and is therefore an estimate of the bilirubin production. During the first days of life, the relationship between the increase in TsB and postnatal age is linear.

The strengths of the study were that the exposed population was homogenous and healthy except for that they were hyperbilirubinemic. In addition, from a methodological standpoint, the neoBLUE radiometer with maximum sensitivity from 440 to $480 \mathrm{~nm}$ matches well with the neoBLUE phototherapy system centered at $458 \mathrm{~nm}$, and the imprecision of the methods for determination of both hemoglobin and TsB was small.

\section{CONCLUSION}

We found that hemoglobin had a significant effect on the efficacy of phototherapy: the higher the hemoglobin concentration, the smaller the decrease in TsB. This knowledge is important for clinicians who treat hyperbilirubinemic infants with phototherapy. These results are compatible with the understanding that the transformation of bilirubin to photobilirubins during phototherapy takes place mainly in the superficial capillaries in the skin. 


\section{Hemoglobin and phototherapy Articles}

\section{ACKNOWLEDGMENTS}

We thank the nurses, doctors in charge, and the laboratory technicians for their excellent assistance.

Disclosure: The authors declare no conflict of interest.

\section{REFERENCES}

1. Maisels MJ. Sister jean ward, phototherapy, and jaundice: a unique human and photochemical interaction. J Perinatol 2015;35:671-5.

2. Ebbesen F, Madsen PH, Vandborg PK, Jakobsen LH, Trydal T, Vreman HJ. Bilirubin isomer distribution in jaundiced neonates during phototherapy with LED light centered at $497 \mathrm{~nm}$ (turquoise) vs. $459 \mathrm{~nm}$ (blue). Pediatr Res 2016;80:511-5.

3. Onishi S, Isobe K, Itoh S, et al. Metabolism of bilirubin and its photoisomers in newborn infants during phototherapy. J Biochem 1986;100:789-95.

4. Lamola AA, Bhutani VK, Wong RJ, Stevenson DK, McDonagh AF. The effect of hematocrit on the efficacy of phototherapy for neonatal jaundice. Pediatr Res 2013;74:54-60.

5. Linfield DT, Lamola AA, Mei E, et al. The effect of hematocrit on in vitro bilirubin photoalteration. Pediatr Res 2016;79:387-90.

6. Ebbesen F, Madsen P, Stovring S, Hundborg H, Agati G. Therapeutic effect of turquoise versus blue light with equal irradiance in preterm infants with jaundice. Acta Paediatr 2007;96:837-41.

7. Ebbesen F, Vandborg PK, Madsen PH, Trydal T, Jakobsen LH, Vreman HJ. Effect of phototherapy with turquoise vs. blue LED light of equal irradiance in jaundiced neonates. Pediatr Res 2016;79:308-12.

8. Doumas BT, Kwok-Cheung PP, Perry BW, et al. Candidate reference method for determination of total bilirubin in serum: development and validation. Clin Chem 1985;31:1779-89.
9. Granati B, Felice M, Fortunato A, Giancola G, Rubaltelli FF. Sites of action of light during phototherapy. Biol Neonate 1983;43:1-8.

10. Donneborg ML, Knudsen KB, Ebbesen F. Effect of infants' position on serum bilirubin level during conventional phototherapy. Acta Paediatr 2010;99:1131-4.

11. Mreihil K, Madsen P, Nakstad B, Benth JS, Ebbesen F, Hansen TW. Early formation of bilirubin isomers during phototherapy for neonatal jaundice: effects of single vs. double fluorescent lamps vs. photodiodes. Pediatr Res 2015;78:56-62.

12. Hansen TW. Therapeutic approaches to neonatal jaundice: an international survey. Clin Pediatr 1996;35:309-16.

13. Bhethanabhotla S, Thukral A, Sankar MJ, Agarwal R, Paul VK, Deorari AK. Effect of position of infant during phototherapy in management of hyperbilirubinemia in late preterm and term neonates: a randomized controlled trial. J Perinatol 2013;33:795-9.

14. Chen CM, Liu SH, Lai CC, Hwang CC, Hsu HH. Changing position does not improve the efficacy of conventional phototherapy. Acta Paediatr 2002;43:255-8.

15. Mohammadzadeh A, Bostani Z, Jafarnejad F, Mazloom R. Supine versus turning position on bilirubin level during phototherapy in healthy term jaundiced neonates. Saudi Med J 2004;25:2051-2.

16. Lamola AA. A pharmacologic view of phototherapy. Clin Perinatol 2016; 43:259-76.

17. Okada H, Masuya K, Yasuda S, et al. Developmental changes in serum half-life of (EZ)-cyclobilirubin. Early Hum Dev 2005;81:619-22.

18. Jopling J, Henry E, Wiedmeier SE, Christensen RD. Reference ranges for hematocrit and blood hemoglobin concentration during the neonatal period: data from a multihospital health care system. Pediatrics 2009; 123:e333-7. 